\title{
Effects of Hall Current on Hydromagnetic Natural Convection Flow of a Heat Absorbing Fluid past an Impulsively Moving Vertical Plate with Ramped Temperature
}

\author{
G. S. Seth ${ }^{1}$, G. K. Mahato ${ }^{1}$, S. Sarkar ${ }^{1}$ and Md. S. Ansari ${ }^{2}$ \\ ${ }^{1}$ Department of Applied Mathematics, Indian School of Mines, Dhanbad-826004 \\ (India) \\ ${ }^{2}$ Faculty of Mathematics, School of Petroleum Technology, PDPU, Gandhinagar- \\ 382007 (India) \\ Email: gsseth_ism@yahoo.com
}

\begin{abstract}
Effects of Hall current on unsteady MHD natural convection flow of a viscous, incompressible, electrically conducting and heat absorbing fluid past an impulsively moving vertical plate with ramped temperature in a porous medium, in the presence of thermal diffusion, is studied. The exact solution of momentum and energy equations, under Boussinesq approximation, is obtained in closed form by Laplace transform technique for both ramped temperature and isothermal plates. Solution is also obtained in the case of unit Prandtl number for ramped temperature plate. The expressions for skin friction due to primary and secondary flows and Nusselt number for both ramped temperature and isothermal plates are also derived. Mathematical formulation of the problem, in non-dimensional form, contains six pertinent flow parameters viz. $M^{2}$ (magnetic parameter), $m$ (Hall current parameter) $G_{r}$ (Grashof number), $K_{1}$ (permeability parameter), $P_{r}$ (Prandtl number) and $\phi$ (heat absorption coefficient). The numerical values of primary and secondary fluid velocities are displayed graphically versus boundary layer coordinate $y$ for various values of $m, G_{r}, K_{1}, \phi$ and time $t$ while that of fluid temperature are presented in graphical form versus $y$ for different values of
\end{abstract}


$P_{r}, \phi$ and $t$ for both ramped temperature and isothermal plates. For both ramped temperature and isothermal plates, the numerical values of skin friction due to primary and secondary flows are presented in tabular form for various values of $m, G_{r}, K_{1}, \phi$ and $t$ whereas that of Nusselt number are given in tables for different values of $P_{r}, \phi$ and $t$.

Keywords: Hall current, Heat absorption coefficient, Natural convection, Ramped temperature, Thermal buoyancy force.

\section{Introduction}

Theoretical/experimental investigation of natural convection flow under the influence of gravitational force past a solid body with different geometries embedded in a fluid-saturated porous medium is of considerable importance due to frequent occurrence of such fluid flow in nature as well as in science and technology viz. geothermal reservoirs, drying of porous solids, thermal insulators, heat exchanger devices, enhanced recovery of petroleum resources, underground energy transport etc. Taking into consideration the importance of such study Cheng and Minkowycz [1] obtained similarity solution for free convection flow from a vertical plate embedded in a fluid-saturated porous medium. Nakayama and Koyama [2] analyzed free and forced convection flow in Darcian and nonDarcian porous medium. Hsieh et al [3] obtained non-similar solution for free and forced convection flow from vertical surfaces in a porous medium. Interest in magnetohydrodynamic convective flow within porous and non-porous media has gained considerable attention of many researchers due to appreciable influence of magnetic field on boundary layer control, thermal insulation of buildings, geothermal energy extraction, plasma studies, enhanced recovery of petroleum resources, sensible heat storage bed and on the performance of many engineering devices using electrically conducting fluids, namely, MHD energy generators, MHD pumps, MHD accelerators, Flow-meters, Plasma jet engines, controlled thermonuclear reactors etc. Raptis and Kafousias [4] investigated influence of magnetic field on steady free convection flow through a porous medium bounded by an infinite vertical plate. Raptis [5] studied mathematically the case of time varying two-dimensional natural convection flow of a viscous, incompressible and electrically conducting fluid past an infinite vertical porous plate embedded in a porous medium. Chamkha [6] analyzed hydromagnetic free convection from an isothermal inclined surface which is near to a thermally stratified porous medium. Sahoo and Sahoo [7] considered MHD free convection and mass transfer flow past an impulsively moving vertical plate through porous medium while Jha [8] discussed this problem when the vertical plate moves with uniform acceleration and applied magnetic field is fixed with the moving plate. Aldoss et al [9] investigated combined free and forced convection flow from a vertical plate embedded in a porous medium in the presence of a magnetic field. Takhar and 
Ram [10] considered hydromagnetic free convection flow of water at $4^{\circ} \mathrm{C}$ through porous medium. Kim [11] investigated hydromagnetic natural convection flow past a vertical moving plate embedded in a porous medium. Ibrahim et al [12] considered unsteady hydromagnetic flow of micro-polar fluid and heat transfer past a vertical porous plate through a porous medium in the presence of thermal and mass diffusion with a constant heat source. Makinde [13] studied MHD mixed convection flow and mass transfer past a vertical porous plate with constant heat flux embedded in a porous medium.

It may be noted that there may be significant temperature difference between the ambient fluid and surface of the solid in so many fluid flow problems of physical interest. This requires the consideration of temperature-dependent heat sources and/or sinks which may have strong influence on heat transfer characteristics (Vajravelu and Nayfeh [14]). Study of the effects of heat generation or absorption in fluid flow is of much significance in several physical problems of practical interest viz. fluids undergoing exothermic and/or endothermic chemical reaction (Vajravelu and Nayfeh [14]), applications in the field of nuclear energy (Crepeau and Clarksean [15]), convection in Earth's mantle (McKenzie et al [16]), post accident heat removal (Baker et al [17]), fire and combustion modeling (Delichatsios [18]), development of metal waste from spent nuclear fuel (Westphal et al [19]) etc. It is noticed that the exact modeling of internal heat generation/absorption is much complicated. It is found that some simple mathematical models yet idealized may express their average behavior for most of the physical situations. Keeping in view of this fact, Sparrow and Cess [20] considered temperature-dependent heat absorption in their research study on steady stagnation point flow and heat transfer. Moalem [21] investigated steady heat transfer in a porous medium with temperature-dependent heat generation. Jha and Ajibade [22] studied free convection flow of heat generating/absorbing fluid within a parallel plate vertical porous channel due to periodic heating of the plates. In their study they considered temperature-dependent heat source/sink. Kamel [23] investigated unsteady hydromagnetic convection flow due to heat and mass transfer through a porous medium bounded by an infinite vertical porous plate with temperature-dependent heat sources/sinks. Chamkha [24] considered unsteady two dimensional convective heat and mass transfer flow of a viscous, incompressible, electrically conducting and temperature-dependent heat absorbing fluid along a semi-infinite vertical permeable moving plate with thermal and concentration buoyancy effects. In all these investigations, analytical/numerical solution is obtained by assuming conditions for the velocity and temperature at the plate as continuous and well defined. However, there are several problems of practical interest which may require non-uniform or arbitrary conditions at the plate. Taking into consideration of this fact, several researchers investigated fluid flow problems of free convection from a vertical plate with step discontinuities in the surface temperature. Mention may be made of the research studies of Hayday et al [25], Kelleher [26], Kao [27], Lee and Yovanovich [28], Chandran et al [29], Seth and Ansari [30] and Seth et al [31]. Seth and Ansari [30] investigated 
unsteady MHD natural convection flow of a viscous, incompressible, electrically conducting and heat absorbing fluid past an impulsively moving vertical plate with ramped temperature in a porous medium taking into account the effects of thermal diffusion. It may be noted that when the density of an electrically conducting fluid is low and/or applied magnetic field is strong (Sutton and Sherman [32]), the effects of Hall current become significant. It plays an important role in determining flow-features of the fluid flow problems because it induces secondary flow in the fluid. Therefore it is of considerable interest to study the effects of Hall current on MHD fluid flow problems. Keeping in view of this fact, Takhar and Ram [33] studied the effects of Hall current on hydromagnetic free convection boundary layer flow of heat generating fluid past a plate in a porous medium using harmonic analysis.

The purpose of present investigation is to study the effects of Hall current on unsteady hydromagnetic natural convection transient flow of a viscous, incompressible, electrically conducting and heat absorbing fluid past an impulsively moving vertical plate embedded in a fluid saturated porous medium, under Boussinesq approximation, taking into account the effects of thermal diffusion when temperature of the plate has a temporarily ramped profile. Natural convection flow resulting from such a plate temperature profile may have bearing on several engineering problems especially where the initial temperature profiles are of much significance in the designing of electromagnetic devices and several natural phenomena which occur due to natural convection and heat generation/absorption.

\section{Formulation of the Problem and its Solution}

Consider unsteady flow of a viscous, incompressible, electrically conducting and temperature-dependent heat absorbing fluid past an infinite vertical plate embedded in a uniform porous medium. Coordinate system is chosen in such a way that $x^{\prime}$ - axis is considered along the plate in upward direction and $y^{\prime}$ - axis normal to the plane of the plate in the fluid. Fluid is permeated by a uniform transverse magnetic field $B_{0}$ which is applied parallel to $y^{\prime}$ - axis. Initially i.e. at time $t^{\prime} \leq 0$, both the fluid and plate are at rest and at a uniform temperature $T_{\infty}{ }^{\prime}$. At time $t^{\prime}>0$, plate starts moving in $x^{\prime}$-direction with uniform velocity $U_{0}$ and temperature of the plate is raised or lowered to $T_{\infty}{ }^{\prime}+\left(T_{w}{ }^{\prime}-T_{\infty}{ }^{\prime}\right) \frac{t^{\prime}}{t_{0}}$ when $t^{\prime} \leq t_{0}$, and thereafter, it is maintained at uniform temperature $T_{w}{ }^{\prime}$. Geometry of the problem is presented in figure 1 . 


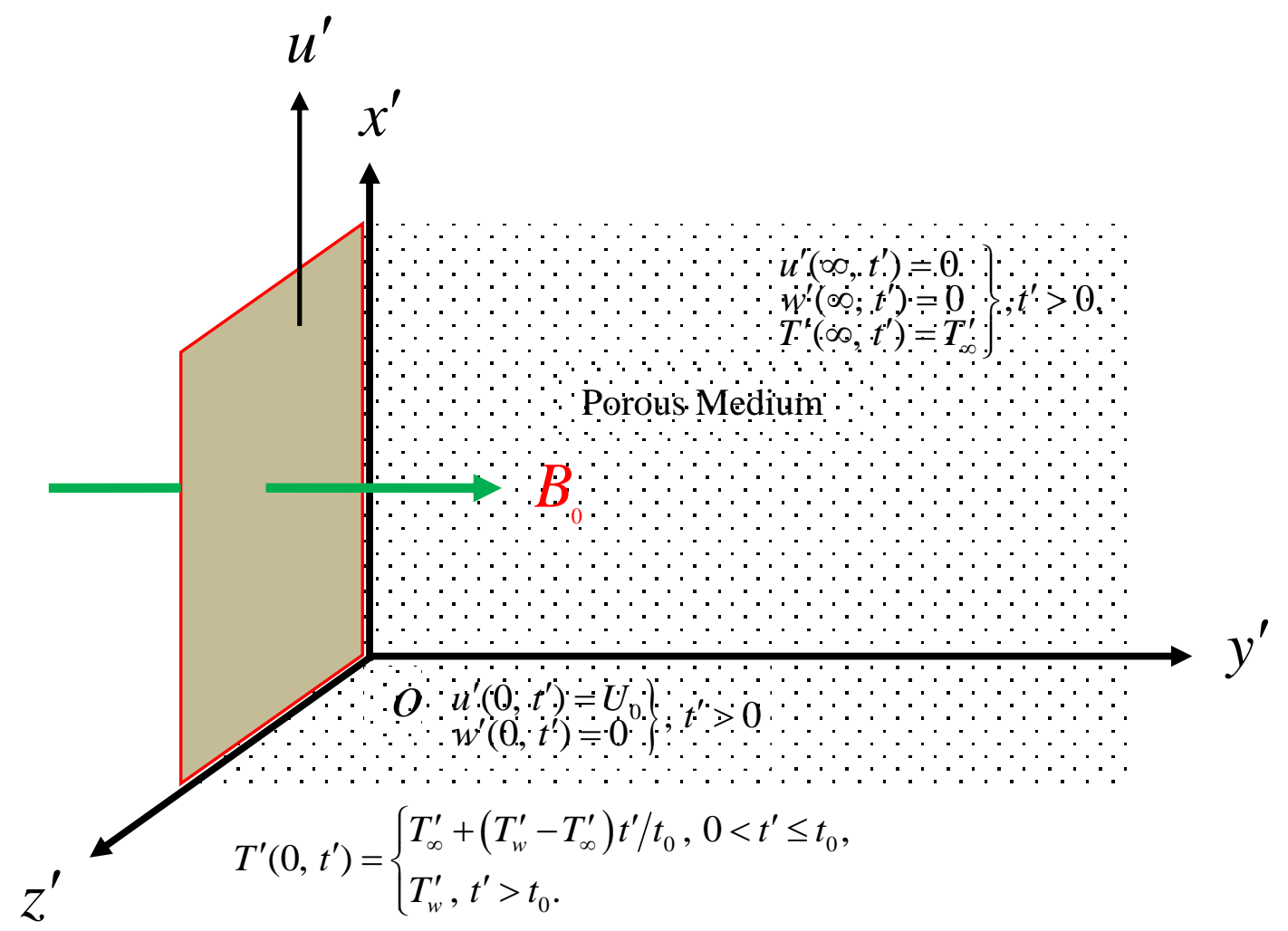

Fig. 1: Geometry of the Problem

Since plate is of infinite extent in $x^{\prime}$ and $z^{\prime}$ directions and is electrically nonconducting all physical quantities, except pressure, depend on $y^{\prime}$ and $t^{\prime}$ only. Also no applied or polarized voltages exist so the effect of polarization of fluid is negligible (Meyer [34]) i.e. $\vec{E} \equiv(0,0,0)$. This corresponds to the case where no energy is added or extracted from the fluid by electrical means. It is assumed that induced magnetic field generated by fluid motion is negligible in comparison to the applied one i.e. $\vec{B} \equiv\left(0, B_{0}, 0\right)$. This assumption is valid because magnetic Reynolds number is very small for liquid metals and partially ionized fluids (Cramer and Pai [35]).

Keeping in view of the assumptions made above, the governing equations for natural convection flow of a viscous, incompressible, electrically conducting and temperature-dependent heat absorbing fluid in a uniform porous medium, under Boussinesq approximation, taking Hall current into account are given by

$$
\frac{\partial u^{\prime}}{\partial t^{\prime}}=v \frac{\partial^{2} u^{\prime}}{\partial y^{\prime 2}}-\frac{\sigma B_{0}{ }^{2}}{\rho\left(1+m^{2}\right)}\left(u^{\prime}+m w^{\prime}\right)-\frac{v}{K_{1}^{\prime}} u^{\prime}+g \beta^{\prime}\left(T^{\prime}-T_{\infty}{ }^{\prime}\right),
$$




$$
\begin{aligned}
& \frac{\partial w^{\prime}}{\partial t^{\prime}}=v \frac{\partial^{2} w^{\prime}}{\partial y^{\prime 2}}+\frac{\sigma B_{0}^{2}}{\rho\left(1+m^{2}\right)}\left(m u^{\prime}-w^{\prime}\right)-\frac{v}{K_{1}^{\prime}} w^{\prime}, \\
& \frac{\partial T^{\prime}}{\partial t^{\prime}}=\frac{k_{1}}{\rho c_{p}} \frac{\partial^{2} T^{\prime}}{\partial y^{\prime 2}}-\frac{Q_{0}}{\rho c_{p}}\left(T^{\prime}-T_{\infty}^{\prime}\right),
\end{aligned}
$$

where $\quad u^{\prime}, w^{\prime}, v, \sigma, \rho, K_{1}^{\prime}, g, \beta^{\prime}, T^{\prime}, T_{\infty}^{\prime}, m=\omega_{e} \tau_{e}, \omega_{e}, \tau_{e}, k_{1}, c_{p} \quad$ and $Q_{0} \quad$ are, respectively, fluid velocity along $x^{\prime}$ - direction, fluid velocity along $z^{\prime}$ - direction, kinematic coefficient of viscosity, electrical conductivity, density, permeability of porous medium, acceleration due to gravity, coefficient of thermal expansion, fluid temperature, temperature in reference state, Hall current parameter, cyclotron frequency, electron collision time, thermal conductivity, specific heat at constant pressure and heat absorption coefficient.

Initial and boundary conditions for the problem are

$$
\left.\begin{array}{l}
u^{\prime}=w^{\prime}=0, T^{\prime}=T_{\infty}^{\prime} \text { for } y^{\prime} \geq 0 \text { and } t^{\prime} \leq 0, \\
u^{\prime}=U_{0}, w^{\prime}=0 \text { at } y^{\prime}=0 \text { for } t^{\prime}>0, \\
T^{\prime}=T_{\infty}^{\prime}+\left(T_{w}^{\prime}-T_{\infty}^{\prime}\right) t^{\prime} / t_{0} \text { at } y^{\prime}=0 \text { for } 0<t^{\prime} \leq t_{0}, \\
T^{\prime}=T_{w}^{\prime} \text { at } y^{\prime}=0 \text { for } t^{\prime}>t_{0}, \\
u^{\prime} \rightarrow 0, w^{\prime} \rightarrow 0, T^{\prime} \rightarrow T_{\infty}^{\prime} \text { as } y^{\prime} \rightarrow \infty \text { for } t^{\prime}>0 .
\end{array}\right\}
$$

Equations (1) to (3), in non dimensional form, assume the form

$$
\begin{aligned}
& \frac{\partial u}{\partial t}=\frac{\partial^{2} u}{\partial y^{2}}-\frac{M^{2}}{\left(1+m^{2}\right)}(u+m w)-\frac{u}{K_{1}}+G_{r} T, \\
& \frac{\partial w}{\partial t}=\frac{\partial^{2} w}{\partial y^{2}}+\frac{M^{2}}{\left(1+m^{2}\right)}(m u-w)-\frac{w}{K_{1}}, \\
& \frac{\partial T}{\partial t}=\frac{1}{P_{r}} \frac{\partial^{2} T}{\partial y^{2}}-\phi T,
\end{aligned}
$$

where

$$
\begin{gathered}
y=y^{\prime} / U_{0} t_{0}, u=u^{\prime} / U_{0}, w=w^{\prime} / U_{0}, t=t^{\prime} / t_{0}, T=\left(T^{\prime}-T_{\infty}^{\prime}\right) /\left(T_{w}^{\prime}-T_{\infty}^{\prime}\right), M^{2}=\sigma B_{0}{ }^{2} v / \rho U_{0}{ }^{2}, \\
K_{1}=K_{1}^{\prime} U_{0}{ }^{2} / v^{2}, G_{r}=g \beta^{\prime} v\left(T_{w}^{\prime}-T_{\infty}^{\prime}\right) / U_{0}{ }^{3}, P_{r}=v \rho c_{p} / k_{1} \text { and } \phi=v Q_{0} / \rho c_{p} U_{0}{ }^{2} .
\end{gathered}
$$


$T, M^{2}, K_{1}, G_{r}, P_{r}$ and $\phi$ are, respectively, non-dimensional fluid temperature, magnetic parameter, permeability parameter, Grashof number, Prandtl number and non-dimensional heat absorption coefficient.

It may be noted that characteristic time $t_{0}$ may be defined, according to the nondimensional process mentioned above, as

$$
t_{0}=v / U_{0}^{2} \text {. }
$$

Initial and boundary conditions (4), in non-dimensional form, become

$$
\begin{aligned}
& u=w=0, T=0 \text { for } y \geq 0 \text { and } t \leq 0, \\
& u=1, w=0 \text { at } y=0 \text { for } t>0, \\
& T=t \text { at } y=0 \text { for } 0<t \leq 1, \\
& T=1 \text { at } y=0 \text { for } t>1, \\
& u \rightarrow 0, w \rightarrow 0 ; T \rightarrow 0 \text { as } y \rightarrow \infty \text { for } t>0 .
\end{aligned}
$$

Combining equations (5) and (6), we obtain

$$
\frac{\partial F}{\partial t}=\frac{\partial^{2} F}{\partial y^{2}}-N F-\frac{1}{K_{1}} F+G_{r} T
$$

where $F=u+i w$ and $N=\frac{M^{2}(1-i m)}{1+m^{2}}$.

Initial and boundary conditions (10a) to (10e), in compact form, are given by

$$
\begin{aligned}
& F=0, T=0 \text { for } y \geq 0 \text { and } t \leq 0, \\
& F=1, \text { at } y=0 \text { for } t>0, \\
& T=t \text { at } y=0 \text { for } 0<t \leq 1, \\
& T=1 \text { at } y=0 \text { for } t>1, \\
& F \rightarrow 0, \quad T \rightarrow 0 \text { as } y \rightarrow \infty \text { for } t>0 .
\end{aligned}
$$

Equations (7) and (11) with the help of Laplace transform technique and initial conditions $(12 \mathrm{a})$ reduce to

$$
\begin{aligned}
& \frac{d^{2} \bar{T}}{d y^{2}}-P_{r}(s+\phi) \bar{T}=0, \\
& \frac{d^{2} \bar{F}}{d y^{2}}-\left(s+N+\frac{1}{K_{1}}\right) \bar{F}+G_{r} \bar{T}=0,
\end{aligned}
$$


where $\bar{F}(y, s)=\int_{0}^{\infty} F(y, t) e^{-s t} d t$ and $\bar{T}(y, s)=\int_{0}^{\infty} T(y, t) e^{-s t} d t, s>0(s$ being the Laplace transform parameter).

Boundary conditions (12b) to (12e), after taking Laplace transform, become

$$
\left.\begin{array}{l}
\bar{F}=1 / s, \quad \bar{T}=\left(1-e^{-s}\right) / s^{2} \text { at } y=0, \\
\bar{F} \rightarrow 0, \quad \bar{T} \rightarrow 0 \text { as } y \rightarrow \infty,
\end{array}\right\} \text {. }
$$

Solving equations (13) and (14) subject to the boundary conditions (15), we obtain

$$
\begin{aligned}
& \bar{T}(y, s)=\frac{\left(1-e^{-s}\right)}{s^{2}} e^{-y \sqrt{P_{r}(s+\phi)}}, \\
& \bar{F}(y, s)=\frac{1}{s} e^{-y \sqrt{s+\lambda}}-\alpha \frac{\left(1-e^{-s}\right)}{s^{2}(s-\beta)}\left\{e^{-y \sqrt{s+\lambda}}-e^{-y \sqrt{P_{r}(s+\phi)}}\right\},
\end{aligned}
$$

where $\alpha=G_{r} /\left(1-P_{r}\right), \lambda=N+\left(1 / K_{1}\right)$ and $\beta=\left(P_{r} \phi-\lambda\right) /\left(1-P_{r}\right)$.

Exact solution for the fluid temperature $T(y, t)$ and fluid velocity $F(y, t)$ is obtained by taking inverse Laplace transform of equations (16) and (17) and is expressed in the following form after simplification (Abramowitz and Stegun [36]).

$$
\begin{aligned}
& T(y, t)=G(y, t)-H(t-1) G(y, t-1), \\
& F(y, t)=\frac{1}{2}\left[e^{y \sqrt{\lambda}} \operatorname{erfc}\left(\frac{y}{2 \sqrt{t}}+\sqrt{\lambda t}\right)+e^{-y \sqrt{\lambda}} \operatorname{erfc}\left(\frac{y}{2 \sqrt{t}}-\sqrt{\lambda t}\right)\right] \\
&-\alpha\left[F^{*}(y, t)-H(t-1) F^{*}(y, t-1)\right],
\end{aligned}
$$

where

$$
G(y, t)=\frac{1}{2}\left[\left(t+\frac{y}{2} \sqrt{\frac{P_{r}}{\phi}}\right) e^{y \sqrt{\phi P_{r}}} \operatorname{erfc}\left(\frac{y}{2} \sqrt{\frac{P_{r}}{t}}+\sqrt{\phi t}\right)+\left(t-\frac{y}{2} \sqrt{\frac{P_{r}}{\phi}}\right) e^{-y \sqrt{\phi P_{r}}} \operatorname{erfc}\left(\frac{y}{2} \sqrt{\frac{P_{r}}{t}}-\sqrt{\phi t}\right)\right],
$$




$$
\begin{aligned}
F^{*}(y, t)= & \frac{1}{2}\left[\frac { e ^ { \beta t } } { \beta ^ { 2 } } \left\{e^{y \sqrt{(\lambda+\beta)}} \operatorname{erfc}\left(\frac{y}{2 \sqrt{t}}+\sqrt{(\lambda+\beta) t}\right)+e^{-y \sqrt{(\lambda+\beta)}} \operatorname{erfc}\left(\frac{y}{2 \sqrt{t}}-\sqrt{(\lambda+\beta) t}\right)\right.\right. \\
& \left.-e^{y \sqrt{P_{r}(\phi+\beta)}} \operatorname{erfc}\left(\frac{y}{2} \sqrt{\frac{P_{r}}{t}}+\sqrt{(\phi+\beta) t}\right)-e^{-y \sqrt{P_{r}(\phi+\beta)}} \operatorname{erfc}\left(\frac{y}{2} \sqrt{\frac{P_{r}}{t}}-\sqrt{(\phi+\beta) t}\right)\right\} \\
& -\frac{1}{\beta}\left\{\left(t+\frac{1}{\beta}+\frac{y}{2 \sqrt{\lambda}}\right) e^{y \sqrt{\lambda}} \operatorname{erfc}\left(\frac{y}{2 \sqrt{t}}+\sqrt{\lambda t}\right)+\left(t+\frac{1}{\beta}-\frac{y}{2 \sqrt{\lambda}}\right) e^{-y \sqrt{\lambda}} \operatorname{erfc}\left(\frac{y}{2 \sqrt{t}}-\sqrt{\lambda t}\right)\right. \\
& \left.-\left(t+\frac{1}{\beta}+\frac{y}{2} \sqrt{\frac{P_{r}}{\phi}}\right) e^{y \sqrt{\phi P_{r}}} \operatorname{erfc}\left(\frac{y}{2} \sqrt{\frac{P_{r}}{t}}+\sqrt{\phi t}\right)-\left(t+\frac{1}{\beta}-\frac{y}{2} \sqrt{\frac{P_{r}}{\phi}}\right) e^{-y \sqrt{\phi P_{r}}} \operatorname{erfc}\left(\frac{y}{2} \sqrt{\frac{P_{r}}{t}}-\sqrt{\phi t}\right)\right\} .
\end{aligned}
$$

$H(t-1)$ and $\operatorname{erfc}(x)$ are, respectively, the unit step function and complementary error function.

\section{Solution in Case of Unit Prandtl Number}

It may be noted that the solution (19) for the fluid velocity is not valid for fluids with unit Prandtl number. Since Prandtl number is a measure of the relative strength of viscosity and thermal conductivity of fluid, then fluid flow problem with $P_{r}=1$ corresponds to those fluids for which both viscous and thermal boundary layer thicknesses are of same order of magnitude. There are some fluids of physical interest which belong to this category (Cebeci [37]). Setting $P_{r}=1$ in Equation (7) and following the same procedure as before, exact solution for fluid temperature $T(y, t)$ and fluid velocity $F(y, t)$ is obtained and is presented in the following form

$$
\begin{aligned}
T(y, t)= & G_{1}(y, t)-H(t-1) G_{1}(y, t-1) \\
F(y, t)= & \frac{1}{2}\left[e^{y \sqrt{\lambda}} \operatorname{erfc}\left(\frac{y}{2 \sqrt{t}}+\sqrt{\lambda t}\right)+e^{-y \sqrt{\lambda}} \operatorname{erfc}\left(\frac{y}{2 \sqrt{t}}-\sqrt{\lambda t}\right)\right] \\
& +\frac{\alpha_{1}}{2}\left[F_{1}(y, t)-H(t-1) F_{1}(y, t-1)\right]
\end{aligned}
$$

where

$$
\begin{aligned}
& \alpha_{1}=G_{r} /(\lambda-\phi) \\
& G_{1}(y, t)=\frac{1}{2}\left[\left(t+\frac{y}{2 \sqrt{\phi}}\right) e^{y \sqrt{\phi}} \operatorname{erfc}\left(\frac{y}{2 \sqrt{t}}+\sqrt{\phi t}\right)+\left(t-\frac{y}{2 \sqrt{\phi}}\right) e^{-y \sqrt{\phi}} \operatorname{erfc}\left(\frac{y}{2 \sqrt{t}}-\sqrt{\phi t}\right)\right],
\end{aligned}
$$




$$
\begin{aligned}
F_{1}(y, t)= & \frac{1}{2}\left[\left(t+\frac{y}{2 \sqrt{\phi}}\right) e^{y \sqrt{\phi}} \operatorname{erfc}\left(\frac{y}{2 \sqrt{t}}+\sqrt{\phi t}\right)+\left(t-\frac{y}{2 \sqrt{\phi}}\right) e^{-y \sqrt{\phi}} \operatorname{erfc}\left(\frac{y}{2 \sqrt{t}}-\sqrt{\phi t}\right)\right. \\
& \left.-\left(t+\frac{y}{2 \sqrt{\lambda}}\right) e^{y \sqrt{\lambda}} \operatorname{erfc}\left(\frac{y}{2 \sqrt{t}}+\sqrt{\lambda t}\right)-\left(t-\frac{y}{2 \sqrt{\lambda}}\right) e^{-y \sqrt{\lambda}} \operatorname{erfc}\left(\frac{y}{2 \sqrt{t}}-\sqrt{\lambda t}\right)\right] .
\end{aligned}
$$

It is observed from the solution (18) that solution (20) for fluid temperature $T(y, t)$ may also be obtained directly by setting $P_{r}=1$ in solution (18).

\section{Solution in Case of Isothermal Plate}

Solutions (18) and (19) represent analytical solution for the fluid temperature and velocity for flow of a viscous, incompressible, electrically conducting and temperature-dependent heat absorbing fluid past an impulsively moving vertical plate with ramped temperature taking Hall current into account. In order to highlight the influence of ramped temperature distribution within the plate on the fluid flow, it may be justified to compare such a flow with the one past an impulsively moving vertical plate with uniform temperature. Keeping in view the assumptions made in this paper, the solution for the fluid temperature and velocity for flow past an impulsively moving isothermal vertical plate is obtained and is presented in the following form

$$
\begin{aligned}
T(y, t) & =\frac{1}{2}\left[e^{y \sqrt{\phi P_{r}}} \operatorname{erfc}\left(\frac{y}{2} \sqrt{\frac{P_{r}}{t}}+\sqrt{\phi t}\right)+e^{-y \sqrt{\phi P_{r}}} \operatorname{erfc}\left(\frac{y}{2} \sqrt{\frac{P_{r}}{t}}-\sqrt{\phi t}\right)\right] \\
F(y, t) & =\frac{\left(1+d^{*}\right)}{2}\left[e^{y \sqrt{\lambda}} \operatorname{erfc}\left(\frac{y}{2 \sqrt{t}}+\sqrt{\lambda t}\right)+e^{-y \sqrt{\lambda}} \operatorname{erfc}\left(\frac{y}{2 \sqrt{t}}-\sqrt{\lambda t}\right)\right] \\
& -\frac{d^{*} e^{\beta t}}{2}\left[\left\{e^{y \sqrt{(\lambda+\beta)}} \operatorname{erfc}\left(\frac{y}{2 \sqrt{t}}+\sqrt{(\lambda+\beta) t}\right)+\left\{e^{-y \sqrt{(\lambda+\beta)}} \operatorname{erfc}\left(\frac{y}{2 \sqrt{t}}-\sqrt{(\lambda+\beta) t}\right)\right\}\right.\right. \\
& \left.-\left\{e^{y \sqrt{P_{r}(\phi+\beta)}} \operatorname{erfc}\left(\frac{y}{2} \sqrt{\frac{P_{r}}{t}}+\sqrt{(\phi+\beta) t}\right)+e^{-y \sqrt{P_{r}(\phi+\beta)}} \operatorname{erfc}\left(\frac{y}{2} \sqrt{\frac{P_{r}}{t}}-\sqrt{(\phi+\beta) t}\right)\right\}\right] \\
& -\frac{d^{*}}{2}\left[e^{y \sqrt{\phi P_{r}}} \operatorname{erfc}\left(\frac{y}{2} \sqrt{\frac{P_{r}}{t}}+\sqrt{\phi t}\right)+e^{-y \sqrt{\phi P_{r}}} \operatorname{erfc}\left(\frac{y}{2} \sqrt{\frac{P_{r}}{t}}-\sqrt{\phi t}\right)\right]
\end{aligned}
$$

where

$d^{*}=G_{r} /\left(1-P_{r}\right) \beta$. 


\section{Skin Friction and Nusselt Number}

Expressions for the primary skin friction $\tau_{x}$, secondary skin friction $\tau_{z}$ and Nusselt number $\mathrm{Nu}$, which are measures of shear stress at the plate due to primary flow, shear stress at the plate due to secondary flow and rate of heat transfer at the plate respectively, are presented in the following form for ramped temperature and isothermal plates.

(i) For ramped temperature plate:

$$
\begin{gathered}
\tau_{x}+i \tau_{z}=\left.\frac{\partial F}{\partial y}\right|_{y=0}=\sqrt{\lambda}(\operatorname{erfc}(\sqrt{\lambda t})-1)-\alpha\left[F_{2}(y, t)-H(t-1) F_{2}(y, t-1)\right] \\
\quad-\frac{1}{\sqrt{\pi t}} e^{-\lambda t}, \\
N u=-\left.\frac{\partial T}{\partial y}\right|_{y=0}=G_{2}(y, t)-H(t-1) G_{2}(y, t-1),
\end{gathered}
$$

where

$$
\begin{aligned}
G_{2}(y, t)= & \frac{1}{2} \sqrt{\frac{P_{r}}{\phi}}(\operatorname{erfc}(\sqrt{\phi t})-1)-t \sqrt{\phi P_{r}}+t\left\{\sqrt{\phi P_{r}} \operatorname{erfc}(\sqrt{\phi t})-\sqrt{\frac{P_{r}}{\pi t}} e^{-\phi t}\right\}, \\
F_{2}(y, t)= & \frac{e^{\beta t}}{\beta^{2}}\left\{\sqrt{(\lambda+\beta)}(\operatorname{erfc}(\sqrt{(\lambda+\beta) t})-1)-\frac{1}{\sqrt{\pi t}} e^{-((\lambda+\beta) t)}\right\} \\
& +\frac{1}{2 \beta \sqrt{\lambda}}(1-\operatorname{erfc}(\sqrt{\lambda t}))+\frac{1}{\beta}\left(t+\frac{1}{\beta}\right)\left\{\sqrt{\lambda}(1-\operatorname{erfc}(\sqrt{\lambda t}))+\frac{1}{\sqrt{\pi t}} e^{-\lambda t}\right\} \\
& -\frac{e^{\beta t}}{\beta^{2}}\left\{\sqrt{P_{r}}(\phi+\beta)(\operatorname{erfc}(\sqrt{(\phi+\beta) t})-1)-\sqrt{\frac{P_{r}}{\pi t}} e^{-((\phi+\beta) t)}\right\} \\
& -\frac{1}{2 \beta} \sqrt{\frac{P_{r}}{\phi}}(1-\operatorname{erfc}(\sqrt{\phi t}))-\frac{1}{\beta}\left(t+\frac{1}{\beta}\right)\left\{\sqrt{\phi P_{r}}(1-\operatorname{erfc}(\sqrt{\phi t}))+\sqrt{\frac{P_{r}}{\pi t}} e^{-\phi t}\right\} .
\end{aligned}
$$

(ii) For isothermal plate:

$$
\begin{aligned}
\tau_{x}+i \tau_{z}= & \left(1+d^{*}\right)\left\{\sqrt{\lambda}(\operatorname{erfc}(\sqrt{\lambda t})-1)-\frac{1}{\sqrt{\pi t}} e^{-\lambda t}\right\}-d^{*} e^{\beta t}\{\sqrt{(\lambda+\beta)}(\operatorname{erfc}(\sqrt{(\lambda+\beta) t})-1) \\
& \left.-\frac{1}{\sqrt{\pi t}} e^{-(\lambda+\beta) t}\right\}-d^{*}\left\{\sqrt{\phi P_{r}}(\operatorname{erfc}(\sqrt{\phi t})-1)-\sqrt{\frac{P_{r}}{\pi t}} e^{-\phi t}\right\} \\
& +d^{*} e^{\beta t}\left\{\sqrt{P_{r}(\phi+\beta)}(\operatorname{erfc}(\sqrt{(\phi+\beta) t})-1)-\sqrt{\frac{P_{r}}{\pi t}} e^{-(\phi+\beta) t}\right\} \\
N u= & \sqrt{\phi P_{r}}(\operatorname{erfc}(\sqrt{\phi t})-1)-\sqrt{\frac{P_{r}}{\pi t}} e^{-\phi t}
\end{aligned}
$$




\section{Results and Discussion}

In order to analyze the effects of Hall current, thermal buoyancy force, permeability of the medium, heat absorption and time on the flow-field, the numerical values of the primary and secondary fluid velocities in the boundary layer region, computed from the analytical solution (19), are displayed graphically versus boundary layer coordinate $y$ for various values of Hall current parameter $m$, Grashof number $G_{r}$, permeability parameter $K_{1}$, heat absorption coefficient $\phi$ and time $t$ taking magnetic parameter $M^{2}=15$ and Prandtl number $P_{r}=0.71$ in figures 2 to 13. Figures 2 to 13 reveal that, for both ramped temperature and isothermal plates, primary velocity $u$ and secondary velocity $w$ attain a distinctive maximum value near the surface of the plate and then decrease properly on increasing boundary layer coordinate $y$ to approach free stream value. Figures 2 to 4 depict the influence of Hall current on the primary velocity $u$ and secondary velocity $w$ for both ramped temperature and isothermal plates. For ramped temperature plate primary velocity $u$ increases while, for isothermal plate, it decreases on increasing Hall current parameter $m$. On the other hand, secondary velocity $w$ increases on increasing $m$ for both the ramped temperature and isothermal plates. This implies that Hall current tends to accelerate fluid flow in the primary flow direction for ramped temperature plate whereas it has reverse effect on the fluid flow in the primary flow direction for isothermal plate. Hall current tends to accelerate fluid flow in the secondary flow direction for both ramped temperature and isothermal plates.

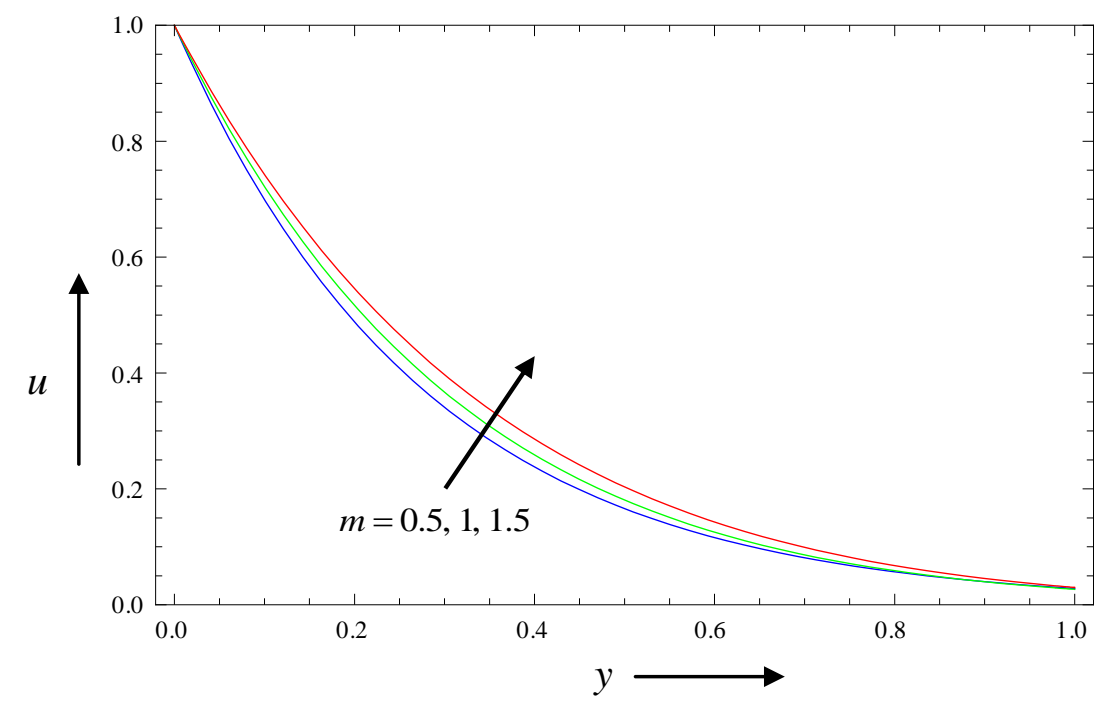

Fig. 2: Primary velocity profiles for ramped temperature plate when $G_{r}=6, K_{1}=0.5, \phi=4$ and $t=0.4$ 


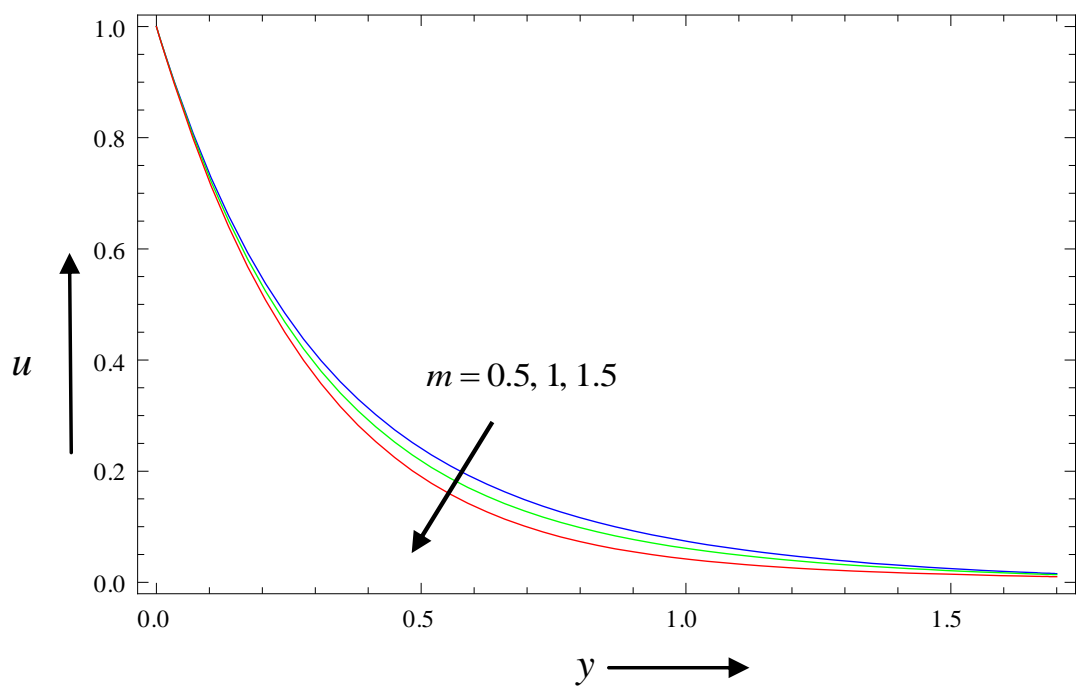

Fig. 3: Primary velocity profiles for isothermal plate when $G_{r}=6, K_{1}=0.5, \phi=4$ and $t=0.4$

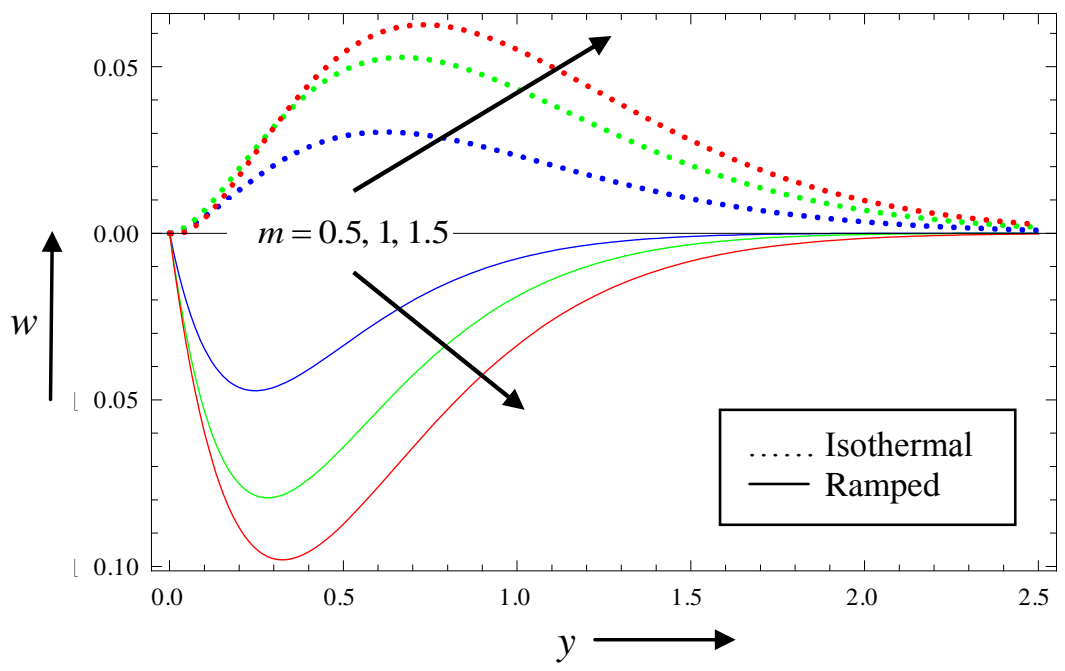

Fig. 4: Secondary velocity profiles when $G_{r}=6, K_{1}=0.5, \phi=4$ and $t=0.4$

Figures 5 and 6 illustrate the effects of thermal buoyancy force on the primary and secondary velocities. As Grashof number increases the primary velocity $u$ increases for both ramped temperature and isothermal plates whereas secondary velocity $w$ decreases for ramped temperature plate and it increases for isothermal plate. This implies that thermal buoyancy force tends to accelerate fluid flow in the primary flow direction for both ramped temperature and isothermal plates. Thermal buoyancy force tends to retard fluid flow in the secondary flow direction for ramped temperature plate and it has reverse effect on the fluid flow in the 
secondary flow direction for isothermal plate. Also there exists reverse flow in the secondary flow direction in the vicinity of isothermal plate when $G_{r}=4$.

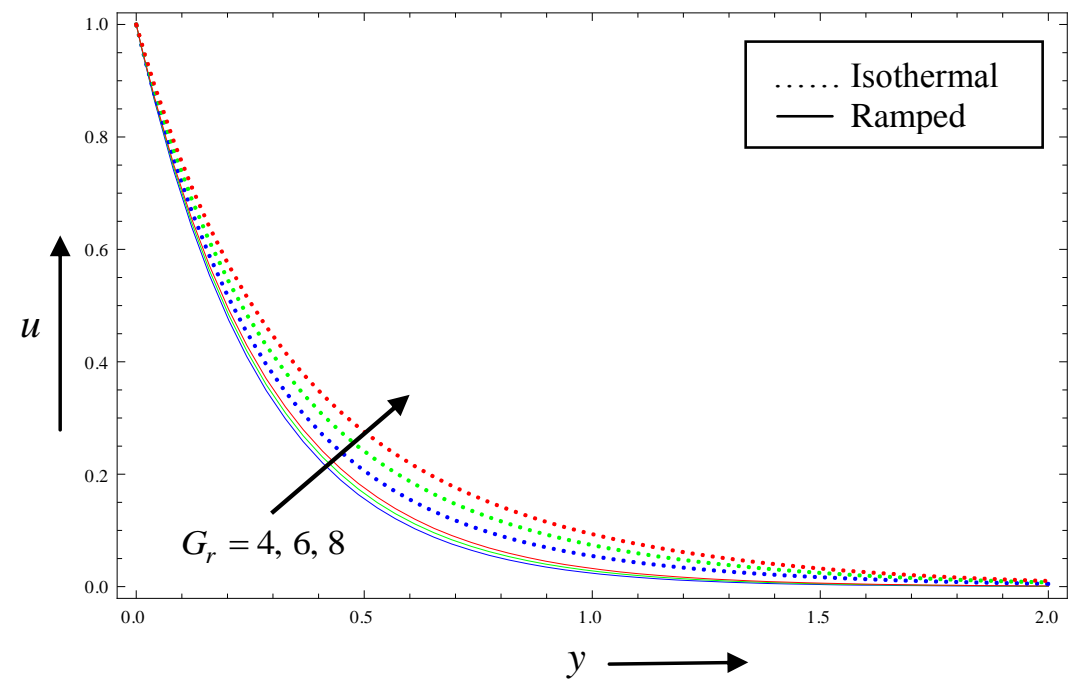

Fig. 5: Primary velocity profiles when $m=0.5, K_{1}=0.5, \phi=4$ and $t=0.4$

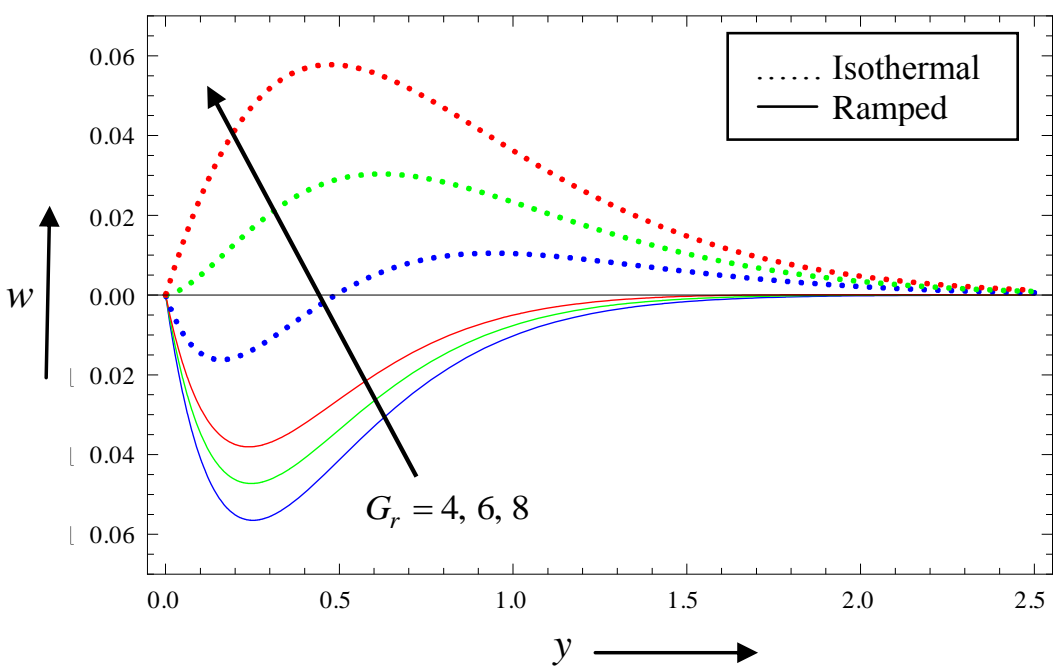

Fig. 6: Secondary velocity profiles when $m=0.5, K_{1}=0.5, \phi=4$ and $t=0.4$

Figures 7 and 8 demonstrate the influence of permeability of porous medium on both primary and secondary velocities. An increase in $K_{1}$, leads to an increase in primary and secondary velocities for both ramped temperature and isothermal plates. This is due to the fact that an increase in $K_{1}$ implies that there is a decrease in the resistance of the porous medium which tends to accelerate fluid flow in both the primary and secondary flow directions for both ramped 
temperature and isothermal plates. Also there exists reverse flow in the secondary flow direction near isothermal plate when $K_{1}=0.2$.

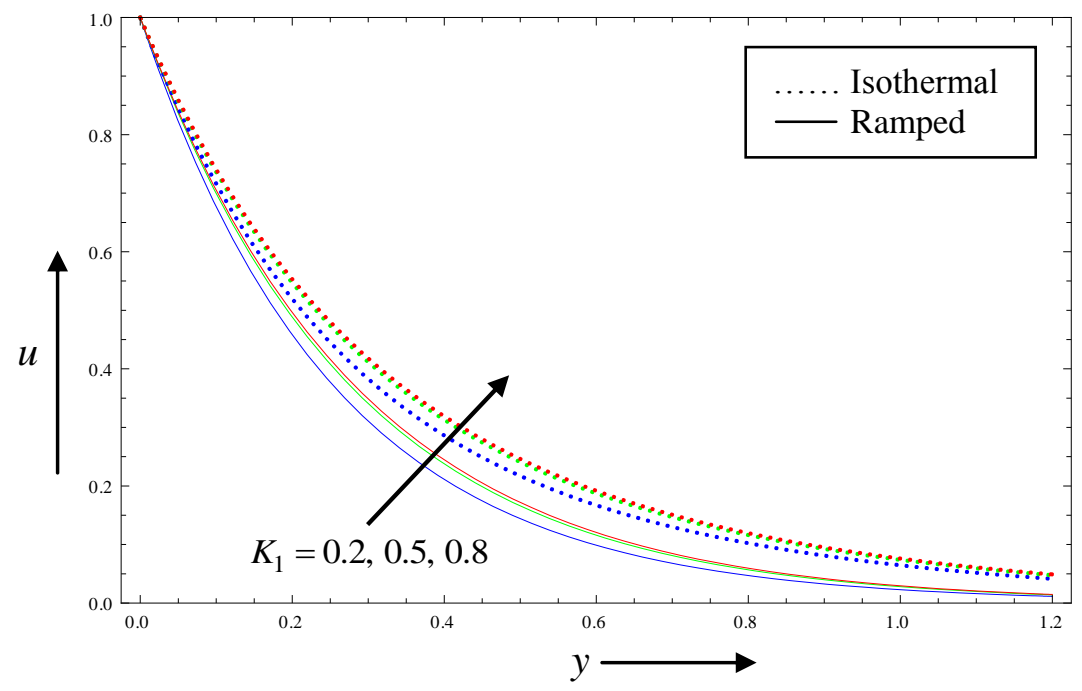

Fig. 7: Primary velocity profiles when $m=0.5, G_{r}=6, \phi=4$ and $t=0.4$

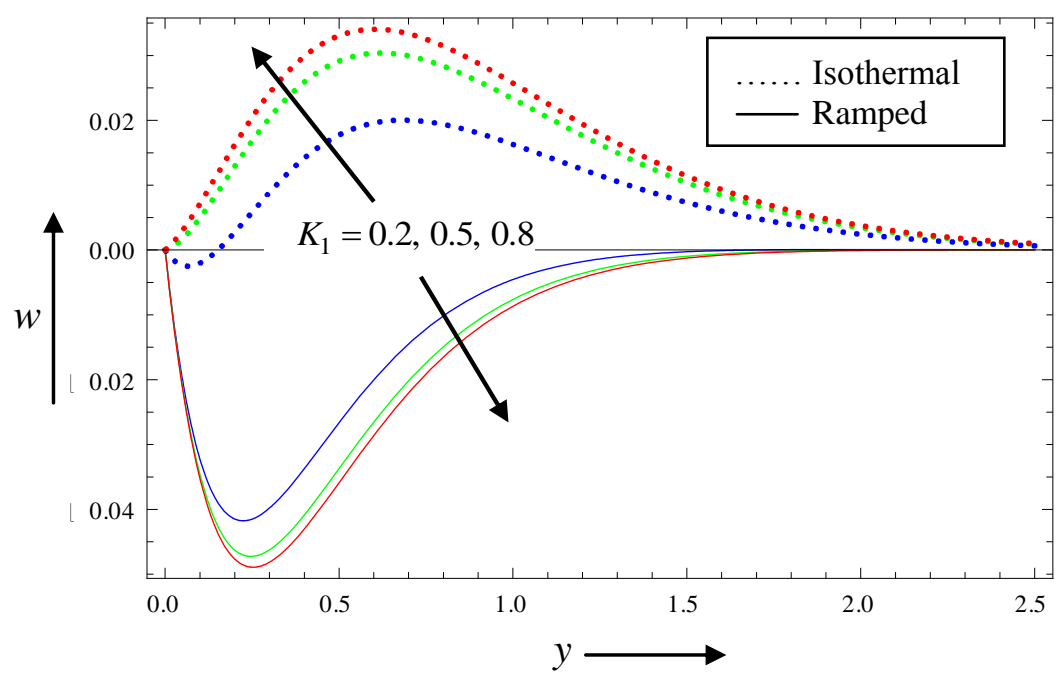

Fig. 8: Secondary velocity profiles when $m=0.5, G_{r}=6, \phi=4$ and $t=0.4$

Figures 9 to 11 display the influence of heat absorption coefficient on the primary and secondary velocities. Primary velocity $u$ decreases on increasing $\phi$ for both ramped temperature and isothermal plates. On increasing $\phi$ secondary velocity $w$ decreases in the vicinity of the plate whereas it increases in the region away from the plate for ramped temperature plate. Secondary velocity $w$ decreases in the region away from the plate on increasing $\phi$ for isothermal plate. This implies that heat absorption tends to retard fluid flow in the primary flow direction for both 
ramped temperature and isothermal plates. Heat absorption tends to retard fluid flow in the secondary flow direction in the vicinity of the plate whereas it has reverse effect on the fluid flow in secondary flow direction in the region away from the plate for ramped temperature plate. For isothermal plate, heat absorption tends to retard fluid flow in the secondary flow direction in the region away from the plate.

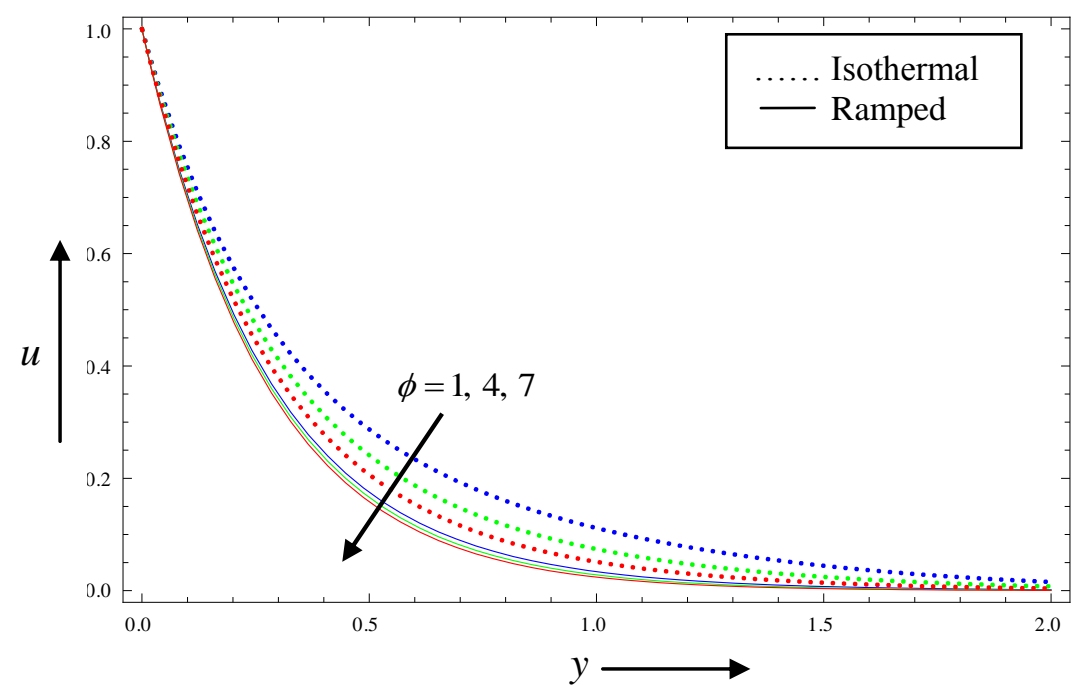

Fig. 9: Primary velocity profiles when $m=0.5, G_{r}=6, K_{1}=0.5$ and $t=0.4$

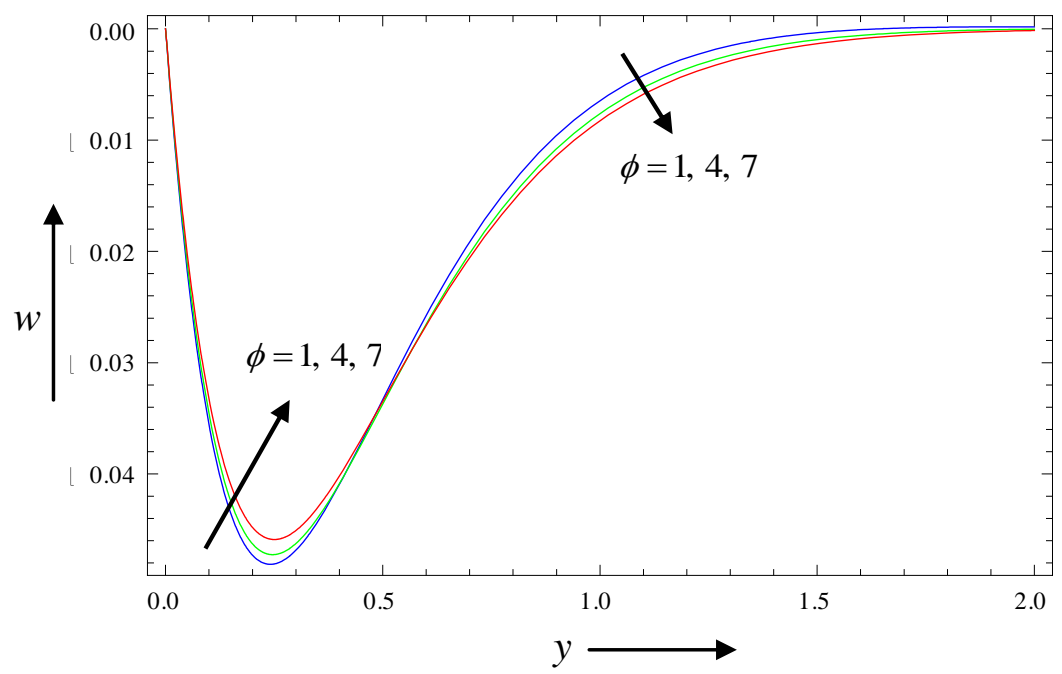

Fig. 10: Secondary velocity profiles for ramped temperature plate when $m=0.5, G_{r}=6, K_{1}=0.5$ and $t=0.4$ 


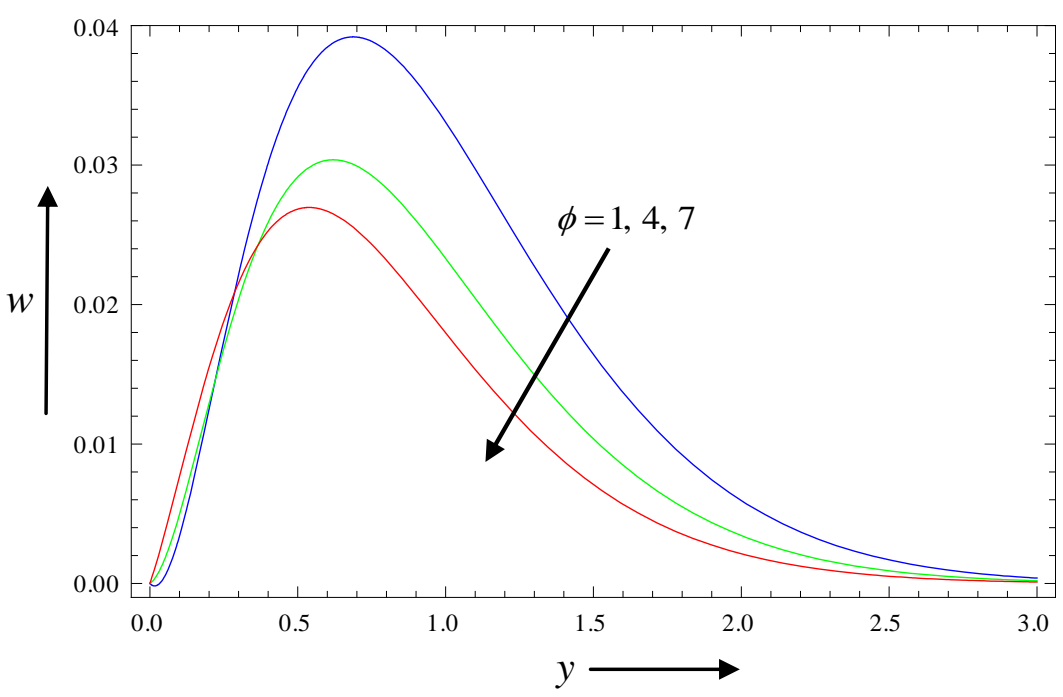

Fig. 11: Secondary velocity profiles for isothermal plate when $m=0.5, G_{r}=6, K_{1}=0.5$ and $t=0.4$

Figures 12 and 13 depict the influence of time on the primary and secondary velocities. As time increases primary velocity $u$ increases for both ramped temperature and isothermal plates whereas secondary velocity $w$ decreases for ramped temperature plate and it increases for isothermal plate. This implies that, as time progresses, there is an enhancement in fluid velocity in the primary flow direction for both ramped temperature and isothermal plates whereas there is a reduction in fluid velocity in the secondary flow direction for ramped temperature plate and an enhancement in fluid velocity in the secondary flow direction for isothermal plate.

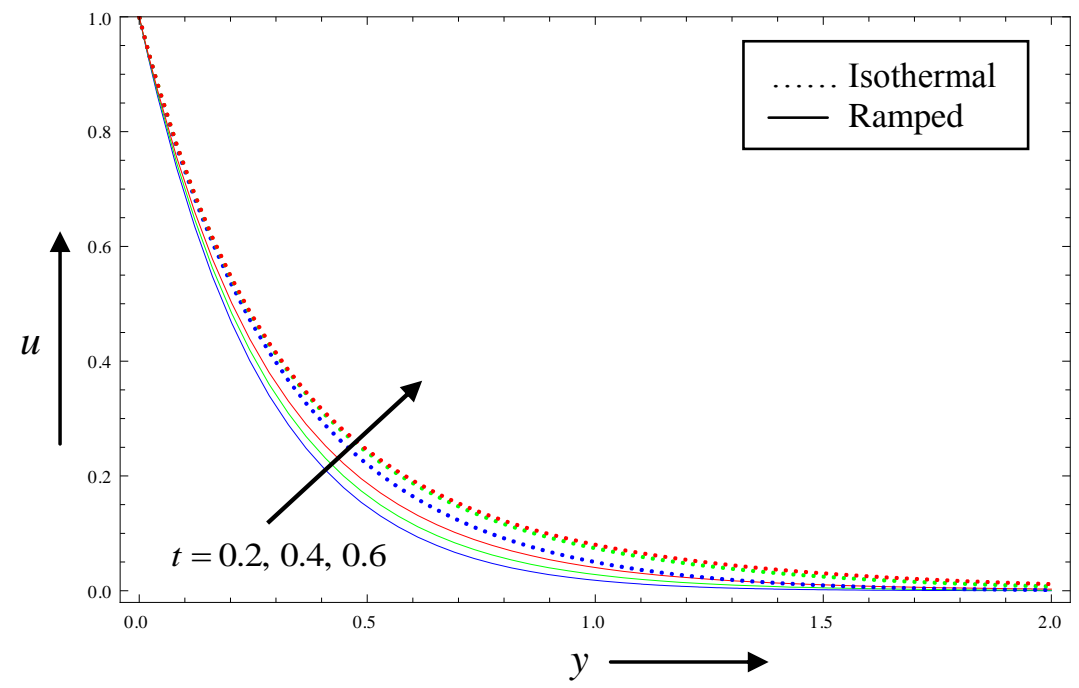

Fig. 12: Primary velocity profiles when $m=0.5, G_{r}=6, K_{1}=0.5$ and $\phi=4$ 


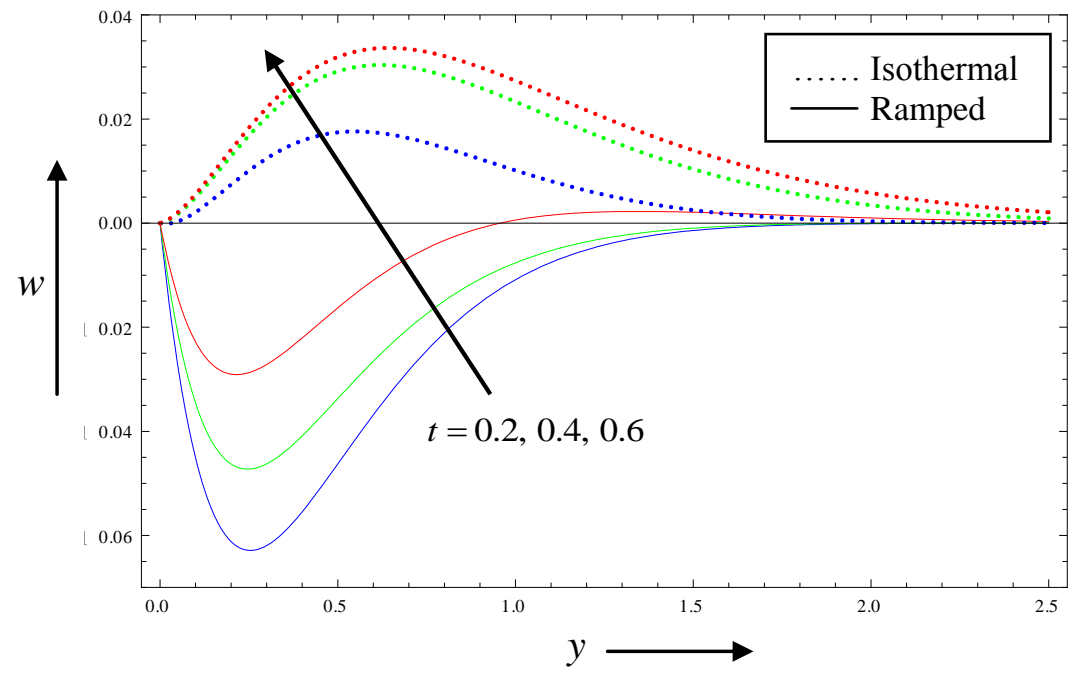

Fig. 13: Secondary velocity profiles when $m=0.5, G_{r}=6, K_{1}=0.5$ and $\phi=4$

The numerical values of fluid temperature $T$, computed from the analytical solution (18), are depicted graphically versus boundary layer coordinate $y$ for various values of Prandtl number $P_{r}$, heat absorption coefficient $\phi$, and time $t$ in figures 14 to 16 .

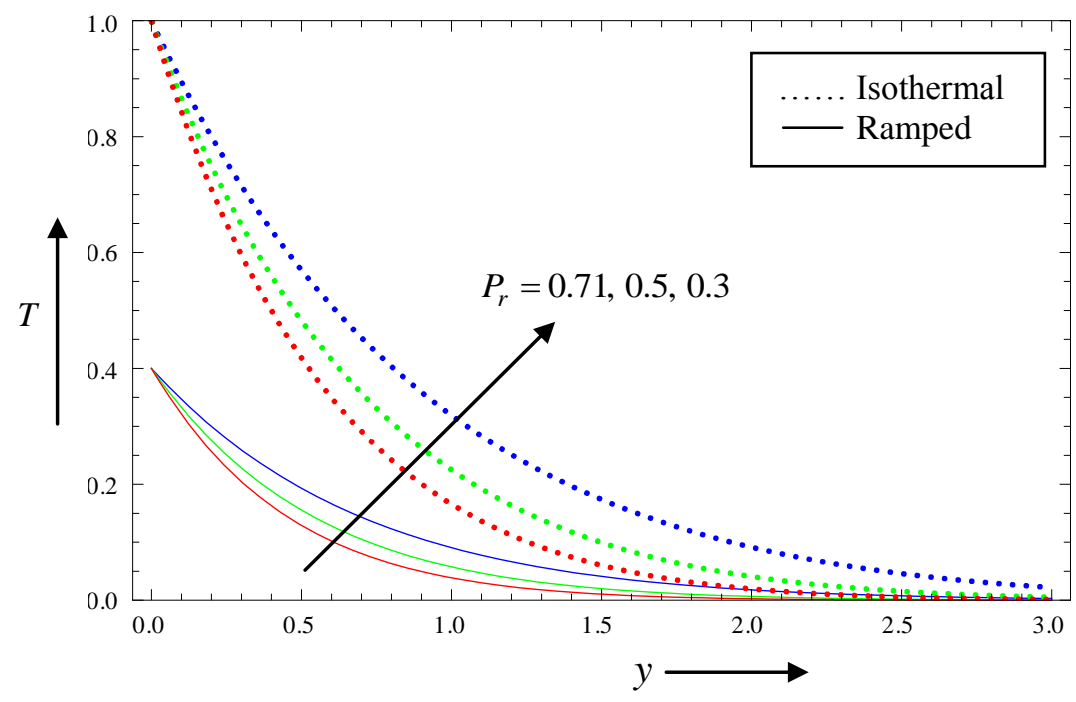

Fig. 14: Temperature profiles when $\phi=4$ and $t=0.4$

Figures 14 to 16 reveal that, for both ramped temperature and isothermal plates, fluid temperature $T$ increases on decreasing Prandtl number $P_{r}$ and it decreases on increasing heat absorption coefficient $\phi$. Fluid temperature $T$ increases on 
increasing time $t . P_{r}$ measures the relative strength of viscosity to thermal conductivity of fluid. $P_{r}$ decreases when thermal conductivity of fluid increases.

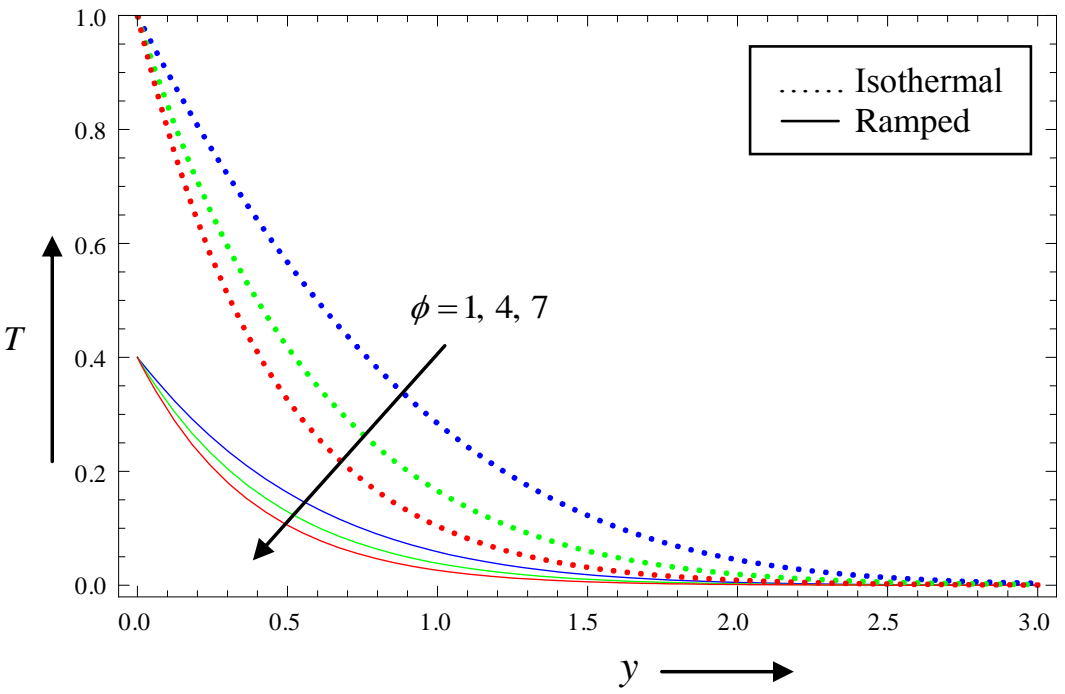

Fig. 15: Temperature profiles when $P_{r}=0.71$ and $t=0.4$

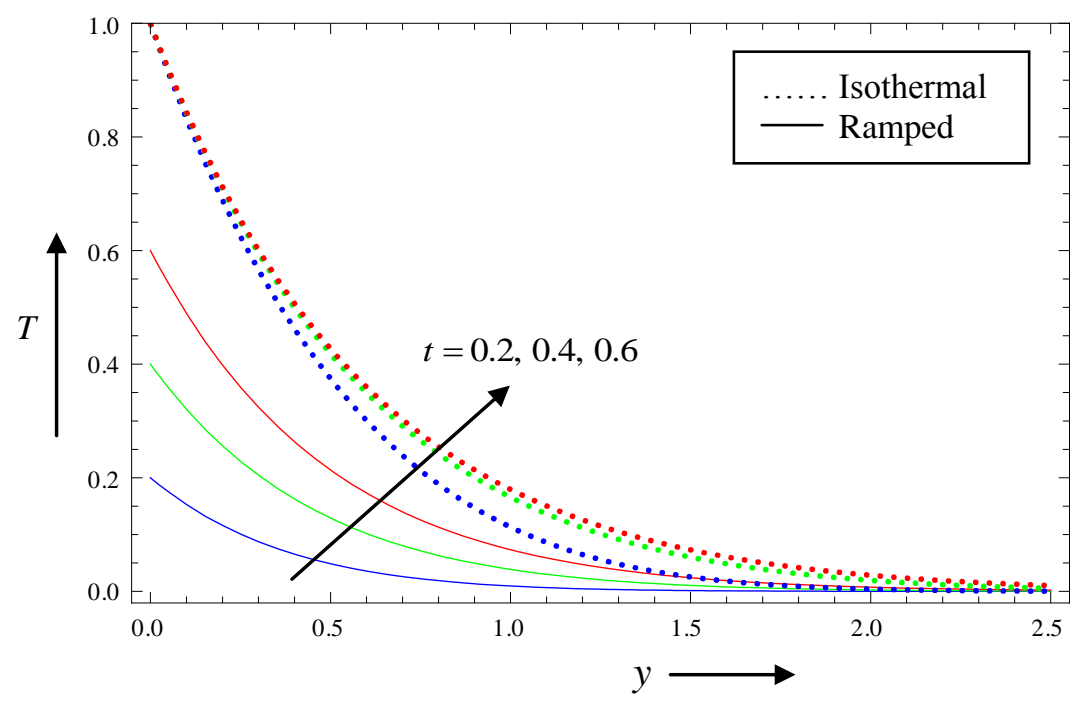

Fig. 16: Temperature profiles when $\phi=4$ and $P_{r}=0.71$

This implies that thermal diffusion tends to enhance fluid temperature whereas heat absorption tends to reduce it for both ramped temperature and isothermal plates. As time progresses, there is an enhancement in fluid temperature for both ramped temperature and isothermal plates. Also fluid temperature is maximum at the surface of the plate for both ramped temperature and isothermal plates and it decreases on increasing boundary layer coordinate $y$ to approach free stream 
value. Also it is evident from figures 14 to 16 that fluid temperature is lower for ramped temperature plate than that of isothermal plate.

The numerical values of non-dimensional skin frictions, $\tau_{x}$ and $\tau_{z}$ due to primary and secondary flow respectively for both ramped temperature and isothermal plates, computed from the analytical expressions (24) and (26), are presented in tabular form in tables 1 to 6 for various values of $m, G_{r}, K_{1}, \phi$ and $t$ taking $M^{2}=15$ and $P_{r}=0.71$ whereas that of Nusselt number $N u$ for both ramped temperature and isothermal plates, computed from analytical expressions (25) and (27), are provided in tables 7 and 8 for different values of $P_{r}, \phi$ and $t$. It is found from table 1 that, for ramped temperature plate, skin friction in primary flow direction i.e. $\tau_{x}$ decreases on increasing $m$ while it decreases on increasing $G_{r}$ when $m \leq 1$. Skin friction in secondary flow direction i.e. $\tau_{z}$ increases on increasing $m$ while it decreases on increasing $G_{r}$. This implies that, for ramped temperature plate, Hall current tends to reduce primary skin friction whereas it has reverse effect on secondary skin friction. On the other hand thermal buoyancy force tends to reduce primary skin friction when $m \leq 1$ while it has same effect on secondary skin friction for every value of $m$.

Table 1: Skin friction for ramped temperature plate when $K_{1}=0.5, \phi=4$ and $t=0.4$

\begin{tabular}{lcccccc}
\hline & \multicolumn{3}{c}{$-\tau_{x}$} & & \multicolumn{3}{c}{$-\tau_{z}$} \\
\cline { 2 - 7 }$m \downarrow G_{r} \rightarrow$ & 4 & 6 & 8 & 4 & 6 & 8 \\
\hline 0.5 & 3.64414 & 3.55478 & 3.46543 & 0.602713 & 0.50953 & 0.416346 \\
1 & 3.23202 & 3.20549 & 3.17896 & 0.873152 & 0.73609 & 0.599029 \\
1.5 & 2.89744 & 2.92667 & 2.95589 & 0.963824 & 0.813587 & 0.66335 \\
\hline
\end{tabular}

Table 2 reveals that, for isothermal plate, $\tau_{x}$ increases on increasing $m$ when $G_{r} \geq 6$ while it decreases on increasing $G_{r} . \tau_{z}$ increases on increasing either $m$ or $G_{r}$. This implies that, for isothermal plate, Hall current tends to enhance primary skin friction when $G_{r} \geq 6$ while thermal buoyancy force has reverse effect on it for every value of $m$. Both the Hall current and thermal buoyancy force have tendency to enhance secondary skin friction. 
Table 2: Skin friction for isothermal plate when $K_{1}=0.5, \phi=4$ and $t=0.4$

\begin{tabular}{|c|c|c|c|c|c|c|}
\hline \multirow[b]{2}{*}{$m \downarrow G_{r} \rightarrow$} & \multicolumn{3}{|c|}{$\tau_{x}$} & \multicolumn{3}{|c|}{$-\tau_{z}$} \\
\hline & 4 & 6 & 8 & 4 & 6 & 8 \\
\hline 0.5 & 2.38391 & 1.66444 & 0.944975 & 0.268394 & 0.797131 & 1.32587 \\
\hline 1 & 2.28857 & 1.79032 & 1.29206 & 0.667822 & 1.57537 & 2.48292 \\
\hline 1.5 & 2.28594 & 2.00942 & 1.73289 & 1.10139 & 2.28424 & 3.46709 \\
\hline
\end{tabular}

It is noticed from table 3 that, for ramped temperature plate, $\tau_{x}$ decreases while $\tau_{z}$ increases on increasing $K_{1}$. This implies that, for ramped temperature plate, permeability of the medium has tendency to reduce primary skin friction whereas it has reverse effect on secondary skin friction.

Table 3: Skin friction for ramped temperature plate when $G_{r}=6, \phi=4$ and $t=0.4$

\begin{tabular}{|c|c|c|c|c|c|c|}
\hline \multirow[b]{2}{*}{$m \downarrow K_{1} \rightarrow$} & \multicolumn{3}{|c|}{$-\tau_{x}$} & \multicolumn{3}{|c|}{$-\tau_{z}$} \\
\hline & 0.2 & 0.5 & 0.8 & 0.2 & 0.5 & 0.8 \\
\hline 0.5 & 3.89425 & 3.55478 & 3.46948 & 0.49448 & 0.50953 & 0.514126 \\
\hline 1 & 3.4932 & 3.20549 & 3.14148 & 0.673763 & 0.73609 & 0.76242 \\
\hline 1.5 & 3.16177 & 2.92667 & 2.88331 & 0.664128 & 0.813587 & 0.887357 \\
\hline
\end{tabular}

It is observed from table 4 that, for isothermal plate, on increasing $K_{1}, \tau_{x}$ decreases when $m=0.5$, it decreases, attains a minimum and then increases when $m=1$ and it increases when $m=1.5 . \tau_{z}$ increases on increasing $K_{1}$. This implies that, for isothermal plate, permeability of the medium has tendency to enhance secondary skin friction. Primary skin friction is of oscillatory nature with respect to permeability of the medium.

Table 4: Skin friction for isothermal plate when $G_{r}=6, \phi=4$ and $t=0.4$

\begin{tabular}{|c|c|c|c|c|c|c|}
\hline \multirow[b]{2}{*}{$m \downarrow K_{1} \rightarrow$} & \multicolumn{3}{|c|}{$\tau_{x}$} & \multicolumn{3}{|c|}{$-\tau_{z}$} \\
\hline & 0.2 & 0.5 & 0.8 & 0.2 & 0.5 & 0.8 \\
\hline 0.5 & 2.15269 & 1.66444 & 1.54853 & 0.448032 & 0.797131 & 0.914434 \\
\hline 1 & 1.87292 & 1.79032 & 1.84229 & 1.01791 & 1.57537 & 1.72533 \\
\hline 1.5 & 1.56226 & 2.00942 & 2.27139 & 1.63248 & 2.28424 & 2.37176 \\
\hline
\end{tabular}


Table 5 illustrates that, for ramped temperature plate, $\tau_{x}$ increases on increasing $\phi$ while it decreases on increasing $t . \tau_{z}$ decreases on increasing either $\phi$ or $t$. This implies that, for ramped temperature plate, heat absorption tends to enhance primary skin friction whereas it has reverse effect on secondary skin friction. Both primary and secondary skin frictions are getting reduced as time progresses.

Table 5: Skin friction for ramped temperature plate when $m=0.5, G_{r}=6$ and $K_{1}=0.5$

\begin{tabular}{lcccccc}
\hline \multirow{2}{*}{$\phi \downarrow t \rightarrow \tau_{x}$} & \multicolumn{3}{c}{$-\tau_{z}$} \\
\cline { 2 - 7 } & 0.2 & 0.4 & 0.6 & 0.2 & 0.4 & 0.6 \\
\hline 1 & 3.64174 & 3.46959 & 3.29878 & 0.780178 & 0.514633 & 0.363237 \\
4 & 3.68077 & 3.55478 & 3.42266 & 0.776461 & 0.50953 & 0.35078 \\
7 & 3.71592 & 3.62626 & 3.53146 & 0.768504 & 0.492907 & 0.322379 \\
\hline
\end{tabular}

It is revealed from table 6 that, for isothermal plate, $\tau_{x}$ decreases on increasing $\phi$ whereas it increases on increasing $t$. On the other hand $\tau_{z}$ increases on increasing $\phi$ while it increases, attains a maximum and then decreases on increasing $t$. This implies that, for isothermal plate, heat absorption has tendency to reduce primary skin friction whereas it has reverse effect on secondary skin friction. Primary skin friction is getting enhanced as time progresses whereas secondary skin friction behaves in oscillatory manner as time progresses.

Table 6: Skin friction for isothermal plate when $m=0.5, G_{r}=6$ and $K_{1}=0.5$

\begin{tabular}{|c|c|c|c|c|c|c|}
\hline \multirow[b]{2}{*}{$\phi \downarrow t \rightarrow$} & \multicolumn{3}{|c|}{$\tau_{x}$} & \multicolumn{3}{|c|}{$-\tau_{z}$} \\
\hline & 0.2 & 0.4 & 0.6 & 0.2 & 0.4 & 0.6 \\
\hline 1 & 1.99005 & 2.05589 & 2.08883 & 0.333701 & 0.367664 & 0.353767 \\
\hline 4 & 1.62832 & 1.66444 & 1.68417 & 0.764136 & 0.797131 & 0.787537 \\
\hline 7 & 1.26088 & 1.26347 & 1.27056 & 1.40266 & 1.43677 & 1.43287 \\
\hline
\end{tabular}

It is evident from tables 7 and 8 that $N u$ increases on increasing either $\phi$ or $P_{r}$ for both ramped temperature and isothermal plates. On increasing $t, N u$ increases for ramped temperature plate while it decreases for isothermal plate. This implies that, for both the ramped temperature and isothermal plates, heat absorption has tendency to enhance rate of heat transfer whereas thermal diffusion has reverse effect on it. As time progresses, rate of heat transfer is getting enhanced for ramped temperature plate while it is getting reduced for isothermal plate. 
Table 7: Nusselt number $-N u$ when $P_{r}=0.71$

\begin{tabular}{lcccccc}
\hline & \multicolumn{3}{c}{ For ramped temperature plate } & \multicolumn{3}{c}{ For isothermal plate } \\
\cline { 2 - 7 }$\phi \downarrow t \rightarrow$ & 0.2 & 0.4 & 0.6 & 0.2 & 0.4 & 0.6 \\
\hline 1 & 0.453002 & 0.678476 & 0.875635 & 1.2688 & 1.03378 & 0.949133 \\
4 & 0.530455 & 0.880298 & 1.22043 & 1.81588 & 1.71289 & 1.69295 \\
7 & 0.600496 & 1.05039 & 1.49675 & 2.28134 & 2.23502 & 2.23019 \\
\hline
\end{tabular}

Table 8: Nusselt number $-N u$ when $\phi=4$

\begin{tabular}{lcccccc}
\hline & \multicolumn{3}{c}{ For ramped temperature plate } & \multicolumn{3}{c}{ For isothermal plate } \\
\cline { 2 - 7 }$P_{r} \downarrow t \rightarrow$ & 0.2 & 0.4 & 0.6 & 0.2 & 0.4 & 0.6 \\
\hline 0.3 & 0.304607 & 0.640004 & 1.06556 & 1.18037 & 1.11343 & 1.10046 \\
0.5 & 0.341191 & 0.679009 & 1.10515 & 1.52385 & 1.43743 & 1.42069 \\
0.71 & 0.372295 & 0.71217 & 1.1388 & 1.81588 & 1.71289 & 1.69295 \\
\hline
\end{tabular}

\section{References}

[1] P. Cheng and W.J. Minkowycz, Free convection about a vertical flat plate embedded in a porous medium with application to heat transfer from a dike, J. Geophys. Res., 82 (1977) 2040-2044.

[2] A. Nakayama and H. Koyama, A general similarity transformation for combined free and forced convection flows within a fluid saturated porous medium, ASME J. Heat Transfer, 109 (1987) 1041-1045.

[3] J.C. Hsieh, T.S. Chen and B.F. Armaly, Non-similarity solutions for mixed convection from vertical surfaces in porous media: variable surface temperature or heat flux, Int. J. Heat Mass Transfer, 36 (1993) 1485-1493.

[4] A. Raptis and N. Kafousias, Heat transfer in flow through a porous medium bounded by an infinite vertical plate under the action of magnetic field, Int. J. Energy Res., 6 (1982) 241-245.

[5] A. Raptis, Flow through a porous medium in the presence of a magnetic field, Int. J. Energy Res., 10 (1986) 97-100.

[6] A.J. Chamkha, Hydromagnetic natural convection from an isothermal inclined surface adjacent to a thermally stratified porous medium, Int. J. Engng. Sci., 35 (1997) 975-986.

[7] D.G. Sahoo and P.K. Sahoo, Hydromagnetic free convection and mass transfer through a porous medium, Ind. J. Tech., 24 (1986) 553-556.

[8] B.K. Jha, MHD free convection and mass-transform flow through a porous medium, Astrophys. Space Sci., 175 (1991) 283-289.

[9] T.K. Aldoss, M.A. Al-Nimr, M.A. Jarrah and B.J. Al-Shaer, Magnetohydrodynamic mixed convection from a vertical plate embedded in a porous medium, Numer. Heat Transfer, 28 (1995) 635-645. 
[10] H.S. Takhar and P.C. Ram, Magnetohydrodynamic free convection flow of water at $4^{\circ} \mathrm{C}$ through a porous medium, Int. Comm. Heat Mass Transfer, 21 (1994) 371-376.

[11] Y.J. Kim, Unsteady MHD convective heat transfer past a semi-infinite vertical porous moving plate with variable suction, Int. J. Engng. Sci., 38 (2000) 833-845.

[12] F.S. Ibrahim, I.A. Hassanien and A.A. Bakr, Unsteady magnetohydrodynamic micro-polar fluid flow and heat transfer over a vertical porous plate through a porous medium in the presence of thermal and mass diffusion with a constant heat source, Canadian J. Phys., 82 (2004) 775790.

[13] O.D. Makinde, On MHD boundary-layer flow and mass transfer past a vertical plate in a porous medium with constant heat flux, Int. J. Numer. Methods Heat Fluid Flow, 19 (2009) 546-554.

[14] K. Vajravelu and J. Nayfeh, Hydromagnetic convection at a cone and a wedge, Int. Comm. Heat Mass Transfer, 19 (1992) 701-710.

[15] J.C. Crepeau and R. Clarksean, Similarity solutions of natural convection with internal heat generation, ASME J. Heat Transfer, 119 (1997) 183-185.

[16] D.P. Mckenzie, J.M. Roberts and N.O. Weiss, Convection in the Earth's mantle: towards a numerical simulation, J. Fluid. Mech., 62 (1974) 465-538.

[17] L. Baker, R.E. Faw and F.A. Kulacki, Post-accident heat removal part-I: heat transfer within an internally heated nonboiling liquid layer, J. Nucl. Sci. Eng., 61 (1976) 222-230.

[18] M.A. Delichatsios, Air entrainment into buoyant jet flames and pool fires in DiNenno, P.J, et al (Eds.) The SFPA handbook of fire protection engineering, NFPA publications, Quincy, M.A., (1988) 306-314.

[19] B.R. Westphal, D.D. Keiser, R.H. Rigg and D.V. Loug, Production of metal waste forms from spent nuclear fuel treatment, DOE Spent Nuclear Fuel Conference, Salt Lake City, UT, (1994) 288-294.

[20] E.M. Sparrow and R.D. Cess, The effect of a magnetic field on free convection heat transfer, Int. J. Heat Mass Transfer, 3 (1961) 267-274.

[21] D. Moalem, Steady state heat transfer with porous medium with temperature dependent heat generation, Int. J. Heat Mass Transfer, 19 (1976) 529-537.

[22] B.K. Jha and A.O. Ajibade, Free convective flow of heat generating/absorbing fluid between vertical porous plates with periodic heat input, Int. Comm. Heat Mass Transfer, 36 (2009) 624-631.

[23] M.H. Kamel, Unsteady MHD convection through porous medium with combined heat and mass transfer with heat source/sink, Energy Conversion and Management, 42 (2001) 393-405.

[24] A.J. Chamkha, Unsteady MHD convective heat and mass transfer past a semi-infinite vertical permeable moving plate with heat absorption, Int. J. Engng Sci., 42 (2004) 217-230. 
[25] A.A. Hayday, D.A. Bowlus and R.A. McGraw, Free convection from a vertical plate with step discontinuities in surface temperature, ASME J. Heat Transfer, 89 (1967) 244-250.

[26] M. Kelleher, Free convection from a vertical plate with discontinuous wall temperature, ASME J. Heat Transfer, 93 (1971) 349-356.

[27] T.T. Kao, Laminar free convective heat transfer response along a vertical flat plate with step jump in surface temperature, Lett. Heat Mass Transfer, 2 (1975) 419-428.

[28] S. Lee and M.M. Yovanovich, Laminar natural convection from a vertical plate with a step change in wall temperature, ASME J. Heat Transfer, 113 (1991) 501-504.

[29] P. Chandran, N.C. Sacheti and A.K. Singh, Natural convection near a vertical plate with ramped wall temperature, Heat Mass Transfer, 41 (2005) 459-464.

[30] G.S. Seth and Md. S. Ansari, MHD natural convection flow past an impulsively moving vertical plate with ramped wall temperature in the presence of thermal diffusion with heat absorption, Int. J. Appl. Mech. Eng., 15 (2010) 199-215.

[31] G.S. Seth, R. Nandkeolyar and Md. S. Ansari, Effect of rotation on unsteady hydromagnetic natural convection flow past an impulsively moving vertical plate with ramped temperature in a porous medium with thermal diffusion and heat absorption, Int. J. Appl. Math. Mech., 7 (2011) 52-69.

[32] G.W. Sutton and A. Sherman, Engineering Magnetohydrodynamics, McGraw-Hill, New York, 1965.

[33] H.S. Takhar and P.C. Ram, Free convection in hydromagnetic flow of a viscous heat-generating fluid with wall temperature oscillation and Hall currents, Astrophys. Space Sci., 183 (1991) 193-198.

[34] R.C. Meyer, Reducing aerodynamic heat transfer rates by magnetohydrodynamic techniques. J. Aero/Space Sci., 25 (1958) 561-572.

[35] K.R. Cramer and S.I. Pai, Magnetofluid dynamics for Engineers and Applied plysicists, McGraw Hill Book Company, New York, 1973.

[36] M. Abramowitz and I.A. Stegun: Handbook of Mathematical Functions, Dover Publications, Inc., New York, 1964.

[37] T. Cebeci, Convective Heat Transfer, Horizons Publishing Inc., Long Beach, California, 2002. 